\title{
Full Duplex 60 GHz Millimeter Wave Transmission over Multi-Mode Fiber
}

\author{
S. R. Abdollahi, H.S. Al-Raweshidy, S. Mehdi Fakhraie*, R. Nilavalan \\ WNCC Research Group, ECE Division, School of Engineering and Design, Brunel University. \\ Uxbridge, Middlesex, UB8 3PH, UK, \\ *University College of Engineering, University of Tehran, North Kargar Ave., Tehran, 14395-515, Iran. \\ E-mail: \{seyedreza.abdollahi, Hamed Al-Raweshidy, Rajagopal.Nilavalan\}@ brunel.ac.uk and fakhraie@ut.ac.ir
}

\begin{abstract}
New wireless subscribers are signing up at an increasing demand of more capacity for ultra-high data rate transfers at speeds more than 1 Gbps, while the radio spectrum is limited. Millimeter wave communication system offers a unique way to resolve these problems. In this paper, the performance of a full duplex transportation system is reported for $1.5 \mathrm{Km}$ of multi-mode fiber length for a sample $10 \mathrm{Gbit} / \mathrm{s}$ pseudo random sequence data, with quadrature amplitude modulation mapping and orthogonal frequency division multiplexing modulation with $60 \mathrm{GHz} \mathrm{RF}$ and coherent $1550 \mathrm{~nm}$ optical carrier. The analysis and simulation results show that the system's quality of service depends on nonlinearity of electro optical modulator, dispersion and signal attenuation impairment of the multimode fiber cable.
\end{abstract}

Keywords - Radio over Fiber, Millimeter Wave, Multi-Mode Fiber, Orthogonal Frequency Division Multiplexing, Quadrature Amplitude Modulation, Electro-Optical Modulator, Laser Source, Central Station and Base Station.

\section{INTRODUCTION}

Wireless communication is entering a new phase where the focus is shifting from voice to multimedia services. Present consumers are no longer interested in the underlying technology, they simply need reliable and cost effective communication systems that can support anytime, anywhere, and any media they want. Furthermore, new wireless subscribers are signing up with an increasing demand of more capacity for ultra-high data rate transfer at speeds of $1 \mathrm{Gbit} / \mathrm{s}$ and more, while the radio spectrum is limited. This requirement of more bandwidth allocation, places heavy burden on the current operating radio frequency (RF) spectrum and causes spectral congestion at lower microwave frequency. Millimeter wave (mm-wave) communication system offers a unique way to resolve these problems, [1]. Mm-wave can be classified as electromagnetic spectrum that spans between 30 to 300 $\mathrm{GHz}$, which corresponds to wavelength from 10 to $1 \mathrm{~mm}$.

In 2001, the Federal Communications Commission (FCC) allocated $7 \mathrm{GHz}$ in the $57-64 \mathrm{GHz}$ band for unlicensed use, [2]. The opening of that free spectrum, combined with the advances of low-cost fabrication technology and low-loss packaging material has revived interest in this portion of wireless spectrum. $60 \mathrm{GHz}$ systems show excellent advantages for the future superbroadband data services delivering superior data rate and power consumption, [3].

For the future provision of broadband, interactive and multimedia services over wireless media, current trends in cellular networks both mobile and fixed are 1) to reduce cell size to accommodate more users and 2) to operate in millimeter wave (mm-wave) frequency band to avoid spectral congestion in lower frequency bands. This demands a large number of base stations (BSs) to cover a service area, and cost effective BS is a key to success in the market. This requirement has led to the development of system architecture where functions such as signal routing/processing, handover and frequency allocation are carried out at a central station (CS), rather than at the BS. An alternative for linking a CS with many BSs in such a radio network is via an optical fiber network, since fiber has low loss, is immune to EMI and has broad bandwidth.

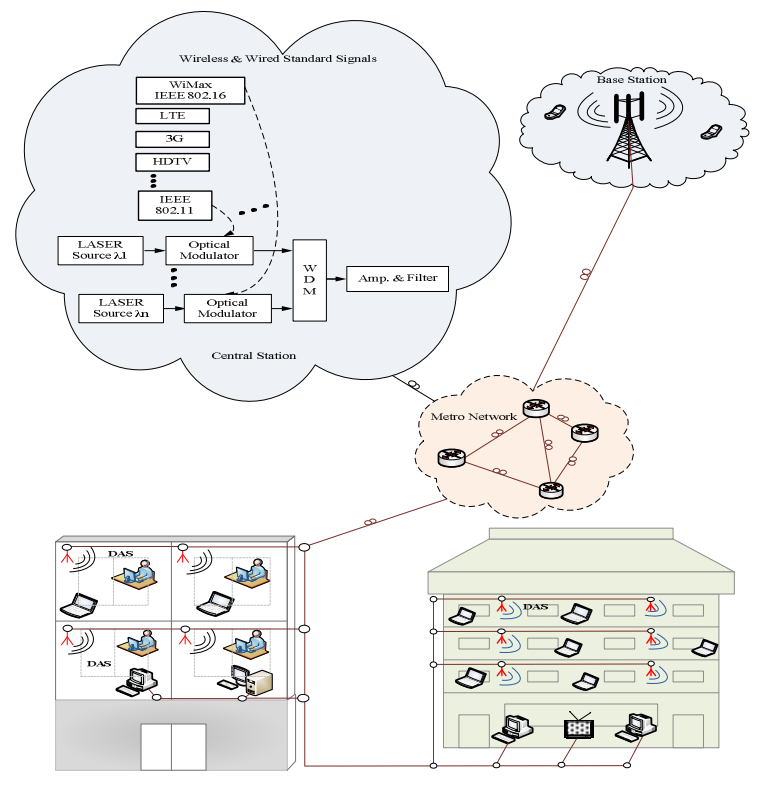

Figure 1. A Radio over Fiber System. 
The technique of modulating the radio frequency (RF) subcarrier onto an optical carrier for distribution over a fiber network is known as "Radio over Fiber" (RoF) technology. RoF technique has been considered a costeffective and reliable solution for the distribution of the future wireless access networks. RoF link is used in remote antenna applications to distribute signals for microcell or picocell BS [4]. In the remote antenna application, the downlink RF signals are distributed from a CS to many BSs known as remote base stations (RBS) through the fibers. The uplink signals received at RBS are sent back to the CS for any signal processing. RoF has the following main features: (1) It is transparent to bandwidth or modulation techniques. (2) Needs simple and small BSs. (3) Centralized operation is possible. The RoF link is illustrated in Fig. 1.

The CSs are connected to RBSs, BSs and fixed customers access network devices through one or two optical fibers, which transport the uplink and downlink signals. The RBSs and BSs act as in-building or green fields picocell or microcell remote antenna units that receive and transmit signals to/from the mobile or fixed users. An RBS is much more cost effective to deploy than a normal BS because it is mostly considered a simple device, which includes Electrical to Optical (E/O) and Optical to Electrical (O/E) converters, Duplexer and an Amplifier.

Furthermore, such a centralized configuration allows sensitive equipment to be located in safer environment and enables the cost of expensive components to be shared among several BSs. Additional to the lower cost advantage, a smaller cell size coverage reduces the near far effect and reduces the transmitted signal power level that is pushed either at the BS/RBS or the mobile transceiver RF power amplifiers. So the proposed wireless access devices consume lower power than traditional cellular access network devices. This enables the users to save more power and use lighter fixed or portable access network devices by using smaller battery, simpler hardware and allows environment friendly systems.

An indoor application of RoF system for wireless access network is shown in Fig. 1. In-building coverage is an important and growing market for wireless and cellular network service providers and operators, who wish to gain and retain customers in environments such as corporate office buildings, shopping malls and airports. The main RoF system architectures that have been used in current commercial in-building wireless deployments are: a) RF transmission over single mode fiber (SMF), b) IF transmission over multi-mode fiber (MMF) or SMF, c) Digitized IF over MMF or SMF.

MMF systems offer flexible, reliable and cost effective cabling solution for local area networks (LANs), central offices, data centers and short range communication networks. MMFs support data rates from 100 megabits per second $(\mathrm{Mb} / \mathrm{s})$ and are in development to $100 \mathrm{~Gb} / \mathrm{s}$. Physically, the MMF has a larger core than SMF, therefore has better coupling efficiency, lower cost and reduces the penalty from the E/O conversion [5].

This paper is organized as follows: Section II, introduces $60 \mathrm{GHz} \mathrm{mm}$-wave radio over fiber system architecture. Section III. provides our system analysis and simulation results. Finally, conclusions are presented in Section IV.

\section{SYSTEM ARCHITECTURE}

In this section, our proposed in-building system architecture is introduced. As shown in Fig. 2, the integrated RoF system includes the CS, RBS and Photonic Distributed Antenna Systems (PDASs). Processing of all transmitted and received signals such as digital, RF and optical signals have been deployed at CS. For distributing the RF and baseband signal over PDASs, some simple signal processing such as multiplexing, demultiplexing, filtering and amplification are implemented at RBS unit. The system is kept more cost effective and is designed based on very simple PDAS, to maintain the performance acceptable for indoor wireless applications.

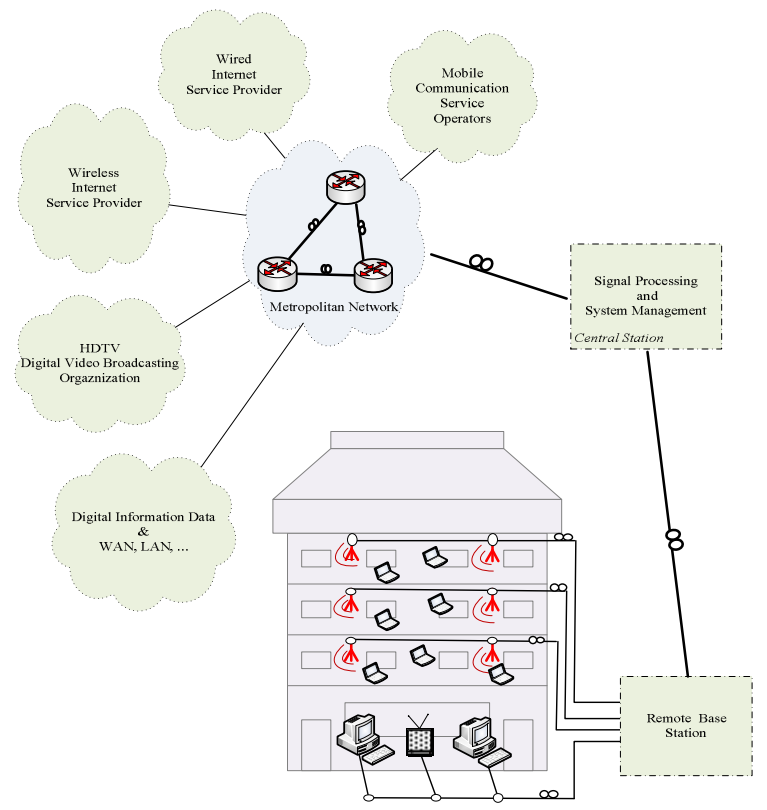

Figure 2. Architecture of indoor Radio over Fiber system.

In this system, the digital information packets are transported from Wide Area Networks (WANs), mobile or fixed Internet Services Providers (ISPs), mobile operators, and digital multimedia broadcasting organizations through metro network to the CS. At the CS, the digital information data from ISPs and digital multimedia broadcasting organizations are transparently transported to the RBS and conveyed to the wired and wireless end-users. The digital information data from wireless and mobile service providers are mapped to Quadrature Amplitude Modulation (QAM) symbols and orthogonally subcarrier modulated by using Orthogonal Frequency Division 
Multiplexing (OFDM) technique. The baseband OFDM signal is up converted to $60 \mathrm{GHz} \mathrm{mm}$-wave band $\mathrm{RF}$ carrier at CS. The generated mm-wave signal is modulated over optical carrier by using Mach-Zehnder Modulator (MZM) electro-optical modulator at $1550 \mathrm{~nm}$ optical source wavelength. By using wavelength division multiplexing (WDM) techniques, the system could be transported to the other wireless standard signals such as 3G, WiMax, LTE, WiFi, and DVB2, concurrently. Therefore, this architecture has been integrated in the photonic system with RF transceiver at RBS and PDAS, for transporting the Analogue RoF (ARoF), Baseband RoF (BRoF), digital (wide area network and LAN) information, and high definition multimedia (HDTV) network data traffics by using the common infrastructure. Therefore, this proposed system has the following features: The ability to support a broad range of services, presenting competition by flexibility, protection of previous and future investments, reliability and low maintenance costs, and seamless upgrade of existing access networks. Therefore, future wireless networks are likely to be integrated with existing optical networks that reduce the future superbroadband mm-wave access network system implementation overheads and service costs to end-users. By implementing this scheme, it will be possible to use free spectrum capacity of metro and access networks for transporting all broadband system traffics such as ARoF and $\mathrm{BRoF}$ and digital information data.

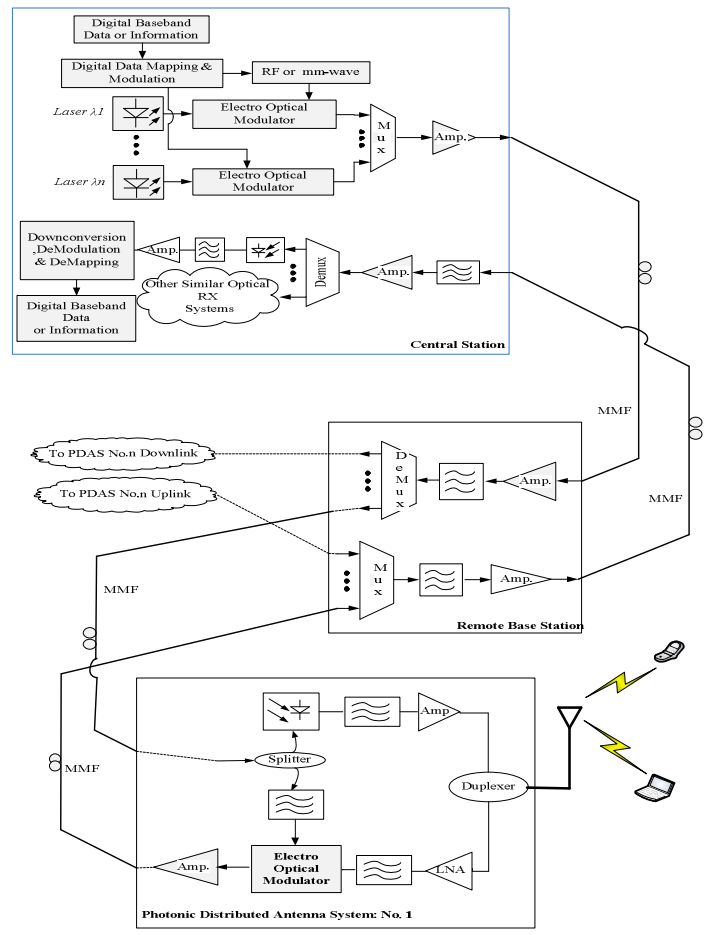

Figure 3. Block diagram of: CS, RBS and PDAS of designed indoor RoF system.

The details of RoF system block diagrams are shown in Fig. 3. The CS, is the central station centralizing and setting up the exclusive and complex signal processing, management and monitoring tasks. This sets up the network more cost-effective and integrated. This system includes transceivers, signal processing, control, and monitoring subsystems. The downlink transmitter includes: the digital baseband signal processing system that performs some tasks such as reframing the packets, and demultiplexing digital information data that are transmitted from metro network. The system transports each type of user's data traffic for performing the mapping and modulation schemes, regarding the particular standard. Finally, the modulated symbols are transmitted by individual optical transmitters such as RF, mm-wave and digital baseband data over fiber. The uplink subsystem that receives the amplified optical signal from RBS, detects the baseband, RF or mm-wave signals and performs the following processes: filtering, amplification, down conversion, detection, demodulation, demapping the symbols, and multiplexing digital information data.

The RBS, which is located nearside the indoor's endusers, is a two way signal repeater that is designed to improve the quality of signal, wavelength multiplexing and demultiplexing, distributing and collecting of the optical signal from or to the uplink and downlink PDASs' subsystem or baseband optical access network systems. The PDAS is simple photonic-wireless integrated transceiver that includes the following blocks: uplink and downlink blocks that receive and transmit the wireless signal from and to the mobile or wireless users. RoF technology makes a distributed antenna system (DAS) further cost effective and reliable. There have been recently many attempts to centralize the light source at the CS and reuse the optical carrier of the downlink signal at the RBS for uplink transmission, [5][6][7]. Therefore, the proposed system cost and circuit's complexity are reduced by reusing the optical carrier at PDASs. In this scheme, the optical carrier is recovered by using very simple splitter and filtered optical carrier out to remove the downlink channels from its spectrum.

\section{ANALYSIS AND SIMULATION}

In this Section, the analysis and simulation results of the proposed system are presented that has been performed by using Optiwave-Optisystem and Matlab environments. The most important block in the broadband $\mathrm{RoF}$ technology is the electro-optical modulator. In this system, the MZM has been used as an electro-optical modulator. The basic MZM is constructed with Lithium Niobate ( $\mathrm{LiNbO} 3$ ) and is a compound of niobium, lithium, and oxygen. Its single crystals provide an important material for optical waveguides, optical modulators and various other linear and non-linear optical applications. Basic structured $\mathrm{LiNbO} 3$ modulator comprises of 1) two waveguides, 2) two Y-junctions, 3) RF/DC electrode. Optical signal coming from the laser diode is lunched into the modulator; it is equally split into two waveguides at first Y-junction on the substrate. When the voltage is not applied to the RF electrode, the two signals are recombined at the second $\mathrm{Y}$-junction and coupled into a single output as two separated signals are inphase. When voltage is applied to the RF electrode, due to electro-optic 
effects of LiNBO3 substrate, refractive index is changed, and phase of the optical signal in one arm is advanced through and retarded in other arm. When two signals are recombined at the second $\mathrm{Y}$-junction, the two signals are out of phase, and the output signal from MZM is recognized as a modulated version of the electrical and optical signals. The power splitting ratio of two arms of balanced MZM is 0.5 [8]. Therefore, the transfer function of MZM is given by

$$
E_{\text {out }}(t)=E_{\text {in }} \cos \left(V(t) \pi / 2 V_{\pi}\right)
$$

where $E_{\text {out }}(t)$ is the electrical modulated field at the output of MZM. $E_{\text {in }}$ is the input optical carrier of laser diode lunched to the MZM, $V(t)$ is applied RF signal voltage and $V_{\pi}$ is the driving voltage [9]. We assume the optical field at the input of the MZM that is lunched from laser diode can be represented by

$$
E_{\text {in }}(t)=E_{o} \cos \left(\omega_{0} \mathrm{t}\right)
$$

where $E_{o}$ and $\omega_{0}$ denote the amplitude and angular frequency of the optical carrier, respectively[6]. Mathematically, each OFDM signal's subcarrier can be described as a complex wave by

$$
S_{c}(t)=A_{c}(t) e^{j\left[\omega_{c} t+\varphi_{c}(t)\right]}
$$

The real signal is the real part of $S_{c}(t)$. Both $A_{c}(t)$ and $\varphi_{c}(t)$, the amplitude and the phase of the carrier can vary on a symbol by symbol basis. The values of the parameters are constant over symbol duration period, $\mathrm{T}$. The OFDM signal consists of $\mathrm{N}$ subcarriers, thus the complex signals $S_{s}(t)$, is represented by

$$
S_{S}(t)=\frac{1}{N} \sum_{n=0}^{N-1} A_{n}(t) e^{j\left[\omega_{n} t+\varphi_{n}(t)\right]}
$$

where $\omega_{n}=\omega_{0}+n \Delta \omega$, this is a continuous signal. Therefore, the OFDM modulated RF signal is the real part of the signal given by equation (4) and is given by

$$
V(t)=\frac{1}{N} \sum_{n=0}^{N-1}\left[A_{n}(t) \cdot \cos \left(\omega_{n} t+\varphi_{n}(t)\right)\right]
$$

Substituting equation (5) in (1), the optical field at the output of modulator is represented by

$E_{\text {out }}(t)=E_{o}\left\{\operatorname{Cos}\left(\omega_{0} \mathrm{t}\right) \cdot \operatorname{Cos}\left(\mathrm{m} \sum_{n=0}^{N-1}\left[A_{n}(t) \cdot \operatorname{Cos}\left(\omega_{n} t+\right.\right.\right.\right.$ $\left.\left.\left.\left.\varphi_{n}(t)\right)\right]\right)\right\}$

where the modulation index $\mathrm{m}$ is $\pi /\left(2 N V_{\pi}\right)$. Expanding the inside element of the braces, by using the Bessel function [10], the output optical field can be rewritten as,

$E_{\text {out }}(t)=E_{o} \cos \left(\omega_{0} \mathrm{t}\right)$

$\times\left[\mathrm{J}_{0}(\mathrm{~m})+2\left(\sum_{\mathrm{k}=1}^{\infty}(-1)^{\mathrm{k}} \mathrm{J}_{2 \mathrm{k}}(\mathrm{m}) \sum_{\mathrm{n}=0}^{\mathrm{N}-1}\left[\mathrm{~A}_{\mathrm{n}}(\mathrm{t}) \cdot \cos \left(\omega_{\mathrm{n}} \mathrm{t}+\right.\right.\right.\right.$ $\left.\left.\left.\left.\varphi_{\mathrm{n}}(\mathrm{t})\right)\right]\right)\right]$

where, $\quad \mathrm{J}_{\mathrm{k}}(\mathrm{m})$ denote the Bessel functions and for any integer $\mathrm{k}$,

$$
\mathrm{J}_{\mathrm{k}}(\mathrm{m})=\frac{1}{\pi} \int_{0}^{\pi} \cos (\mathrm{m} \cdot \sin \theta-\mathrm{k} \theta) \mathrm{d} \theta
$$

Therefore, equation (7) can be given by

$$
\begin{array}{r}
E_{\text {out }}(t)=E_{o} \mathrm{~J}_{0}(\mathrm{~m}) \cos \left(\omega_{0} \mathrm{t}\right)+ \\
\sum_{\mathrm{k}=1}^{\infty}\left\{(-1)^{\mathrm{k}} \mathrm{J}_{2 \mathrm{k}}(\mathrm{m}) \cdot \sum_{n=0}^{N-1} A_{n}(t) \times\right. \\
\left.\left[\begin{array}{c}
\cos \left(\left(\omega_{0}+\omega_{n}\right) \mathrm{t}+\varphi_{n}(t)\right)+ \\
\cos \left(\left(\omega_{0}-\omega_{n}\right) \mathrm{t}-\varphi_{n}(t)\right)
\end{array}\right]\right\}
\end{array}
$$

The first term of equation (9) is the optical carrier and the second term discuses the sidebands of RF or mm-wave signal. As represented in this equation, the nonlinearity of MZM modulator produces infinite terms of signal spurious band that affects the system performance and the quality of the service (QoS) of RoF system. In this model, the one kilometer multi-mode fiber is routed between the CS and RBS and the fiber cable length between RBS to PDAS was about five hundred meters. Fig. 4 shows the simulated transmitted optical spectrum.

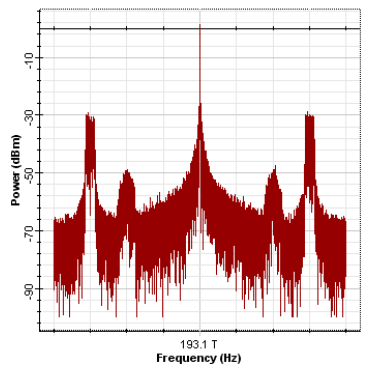

(a)

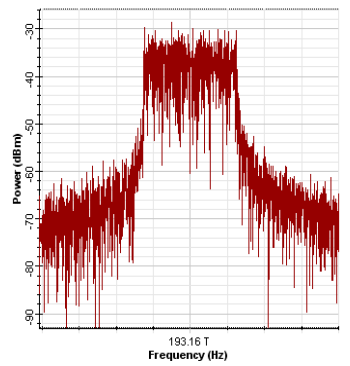

(b)
Figure 4. Spectrum of simulated 4QAM-OFDM signal with $60 \mathrm{GHz}$ carrier (a) Optical carrier spectrum, (b) OFDM optical signal spectrum.

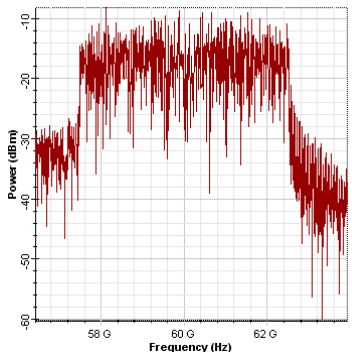

(a)

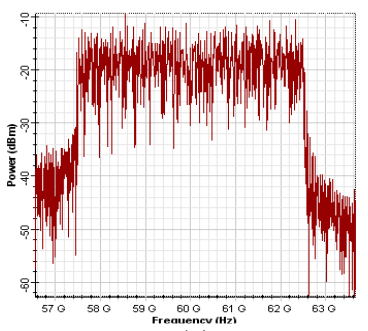

(c)

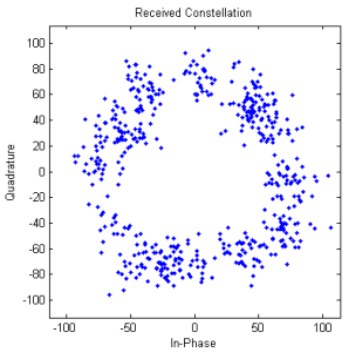

(b)

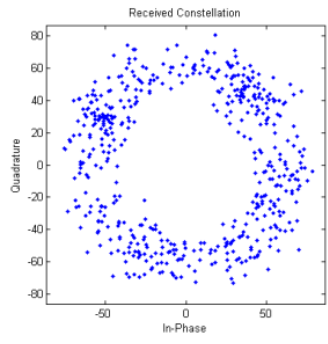

(d)
Figure 5. Downlink received signal, (a) Spectrum, (b) Constellation, and Uplink received signal, (c) Spectrum, (d) Constellation, over $1.5 \mathrm{Km}$ $\mathrm{MMF}$ 
In this system simulation, the attenuation and dispersion of the MMF are assumed $2 \mathrm{~dB} / \mathrm{Km}$ and 100 $\mathrm{ps} / \mathrm{nm} / \mathrm{km}$, respectively, and a $10 \mathrm{~mW}$ laser source at 1550 $\mathrm{nm}$ is used at CS as a continuous wave optical source. The system transports full-duplex 10Gbit/s pseudo random sequence that is OFDM modulated with 4QAM mapping over $60 \mathrm{GHz} \mathrm{RF}$ carrier that is modulated on optical carrier.

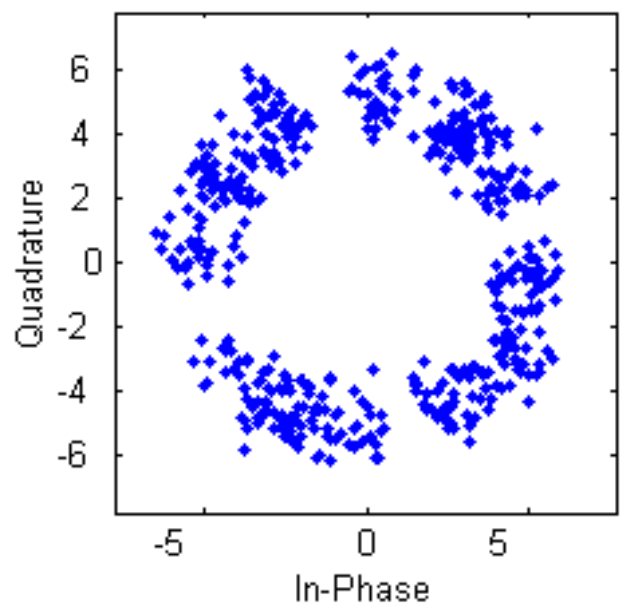

(a)

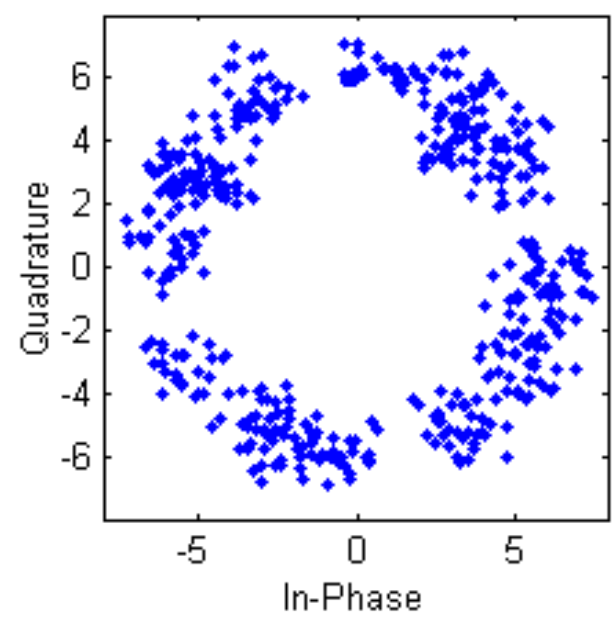

(b)

Figure 6. received signal, (a): Downlink Constellation, and (b): Uplink Constellation, over 1.2 Km MMF .

The system performance is shown in Fig. 5 for fullduplex RoF signal transportation over $1.5 \mathrm{Km}$ of $\mathrm{MMF}$ length and Fig. 6 shows the system performance over 1.2 $\mathrm{Km}$ of MMF cable length that shows the performance severely depends on the fiber attenuation, dispersion impairment.

\section{CONCLUSIONS}

In this paper, the proposed full duplex transmission of $60-\mathrm{GHz} \mathrm{mm}$-wave band radio signal over MMF for inbuilding wireless communication services is simulated by using Optiwave-Optisystem and Matlab simulation tools. This advanced technology is designed to satisfy both mobile and fixed users for the future provision of broadband and interactive multimedia services and current trends in cellular networks. The performance of the proposed RoF system has been investigated and simulated for about $1.5 \mathrm{Km}$ of MMF cable length. The attenuation of cable is assumed $2 \mathrm{~dB} / \mathrm{Km}$ and the dispersion 100 $\mathrm{ps} / \mathrm{nm} / \mathrm{Km}$. The simulation results show that the system's QoS is dependent on the modulator performance and the passive optical communication devices. The dispersion and signal attenuation impairments of the MMF directly affect the QoS. The performance of the full duplex system is reported for $1.5 \mathrm{Km}$ of fiber length for transporting a sample $10 \mathrm{Gbit} / \mathrm{s}$ pseudo random sequence, with 4QAM mapping and OFDM modulation.

\section{REFERENCES}

[1] Kaszubowska, L. H, and L. P. Barry, "Remote down conversion with wavelength reuse for the radio/fiber uplink connection", IEEE Photonics Technology Letters, vol. 18, no. 4, Feb. 2006.

[2] N. Guo, R. Qiu, S. Mo, K. Takahashi, " 60 GHz millimeter-wave radio: principle, technology, and new results," EURASIP Journal of Wireless Communication and Networking, no 68253, vol. 2007 , pp. 1-8, 2007.

[3] J. Laskar, S.Pinel, D. Dawn, S. sarkar, B. Perumana and P. sen, “ The next wireless wave is a millimeter wave," Microwave Journal,vol 90, no. 8, pp 22-35, August 2007.

[4] H.S. Al-Raweshidy and S. Komaki, "Radio over fiber technologies for mobile communication network", Artech House, 2002.

[5] J. Yu., Z. Jia, T. Wang, and G-K Chang, “ A novel radio-over-fiber configuration using optical phase modulator to generate an optical mm-wave and centralized lightwave for uplink connection," IEEE Photon. Technol. Lett., vol. 19, no. 4, pp. 140-142, Feb. 2007.

[6] J. J. V. Olmos, T, Kuri, and K. I. Kitayama, "60 GHz-band 155 $\mathrm{Mb} / \mathrm{s}$ and $1.5 \mathrm{~Gb} / \mathrm{s}$ baseband time slotted full-duplex radio-overfiber access network," IEEE Photo. Technol. Lett., vol. 20, no. 7, pp. 617-619, Apr. 2008

[7] L. Chen, H. Wen, and S. Wen, " A radio-over-fiber system with a novel scheme for millimeter-wave generation and wavelength reuse for uplink connection," IEEE Photon, Technol. Letter., vol. 18, no. 19, pp. 2056-2058, Oct. 2006.

[8] Charles H. Cox, III, "Analog Optical Links Theory and Practice", Cambridge press, 2004.

[9] Chun-Ting Lin, Jason Chen, Sheng-Peng Dai, Peng-Chun Peng, and Sien Chi, "Impact of nonlinear transfer function and impact splitting ratio of MZM on optical up-conversion employing double sideband with carrier suppression modulation", Journal of Lightwave Technology, vol. 26, no. 15, pp. 2449-2459, Aug. 2008

[10] B. G. Korenev, Bessel Functions and Their Applications, Taylor and Francis, 2002. 